Sonderdruck aus

\title{
Archiv der Pharmazie
}

303. Band, Heft 2, Seite 145-148

Verlag Chemie, GmbH, Weinheim/Bergstr.

W. Wiegrebe und W. D. Sasse

Synthese und Hofmann-Abbau des 1-Hydroxymethyl-2,2dimethyl-1,2,3,4-tetrahydroisochinolinium-Ions 


\section{.}


W. Wiegrebe und W. D. Sasse

\section{Synthese und Hofmann-Abbau des 1-Hydroxymethyl-2,2- dimethyl-1,2,3,4-tetrahydroisochinolinium-Ions}

Aus dem Institut für Pharmazeutische Technologie der Technischen Universität Braunschweig

(Eingegangen am 7. Mai 1969)

Analog zu den Derivaten des 1- $\alpha$-Hydroxybenzyl-2,2-dimethyl-1,2,3,4-tetrahydroisochinolinium-Ions entstehen aus dem 1-Hydroxymethyl-2,2-dimethyl-1,2,3,4-tetrahydroisochinolinium-Ion unter den Bedingungen des Hofmann-Abbaus N,N-Dimethyl-2-vinyl-benzylamin und Formiat.

\section{Synthesis and Hofmann-Degradation of 1-Hydroxymethyl-2,2-dimethyl-1,2,3,4- tetrahydroisoqinolinium-Ion}

Analogous to the derivatives of the 1- $\alpha$-hydroxybenzyl-2,2-dimethyl-1,2,3,4-tetrahydroisoquinolinium-ion, $\mathrm{N}, \mathrm{N}$-dimethyl-2-vinyl-benzylamine and formate arise from the 1-hydroxymethyl-2,2-dimethyl-1,2,3,4-tetrahydroisoquinolinium-ion under the conditions of Hofmann-degradation.

Beim Hofmann-Abbau entstehen aus der quartären Base des 1- $\alpha$-Hydroxybenzyl2-methyl-1,2,3,4-tetrahydroisochinolins (I) Benzaldehyd und N,N-Dimethyl-2-vinylbenzylamin (II); durch die Einwirkung der Lauge reagiert Benzaldehyd zu Benzoesäure $\left.{ }^{\mathbf{1}}\right)$. Die Reaktion läuft über eine Ylid-Zwischenstufe $\left.(\mathrm{III})^{2}\right)^{3}$ ).

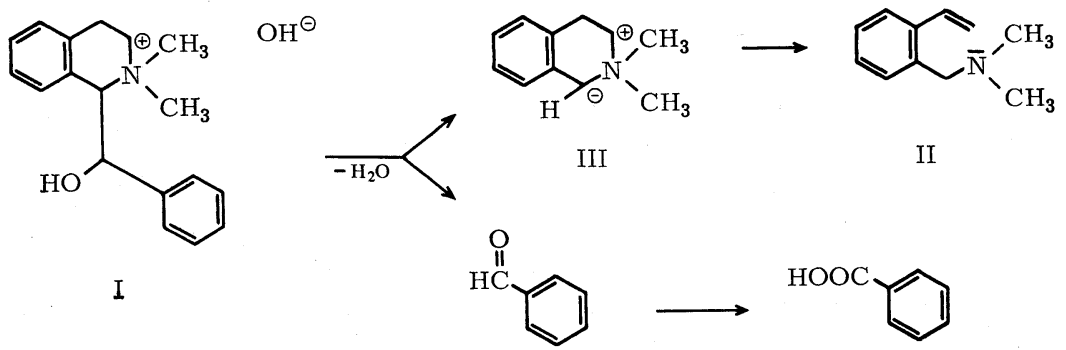

Bisher untersuchten wir das Reaktionsverhalten sekundärer Alkohole vom Typ I. Es war daher wünschenswert zu erfahren, ob auch bei primären Carbinolen der Substituent an C-1 unter den Bedingungen des Hofmann-Abbaus abgespalten wird.Das 1-Hydroxymethyl-2,2-dimethyl-1,2,3,4-tetrahydroisochinolinium-Ion (IV, s. Formelübersicht) ist die einfachste Verbindung, an der diese Frage geprüft werden kann. Wir haben daher IV nach dem in der Übersicht dargestellten Schema synthetisiert:

1) W. Wiegrebe und W. Awe, Arch. Pharmaz. 296, 807 (1963).

2) W. Wiegrebe, Arch. Pharmaz. 296, 801 (1963).

3) W. Wiegrebe und W. Awe, Naturwissenschaften 49, 325 (1962). 


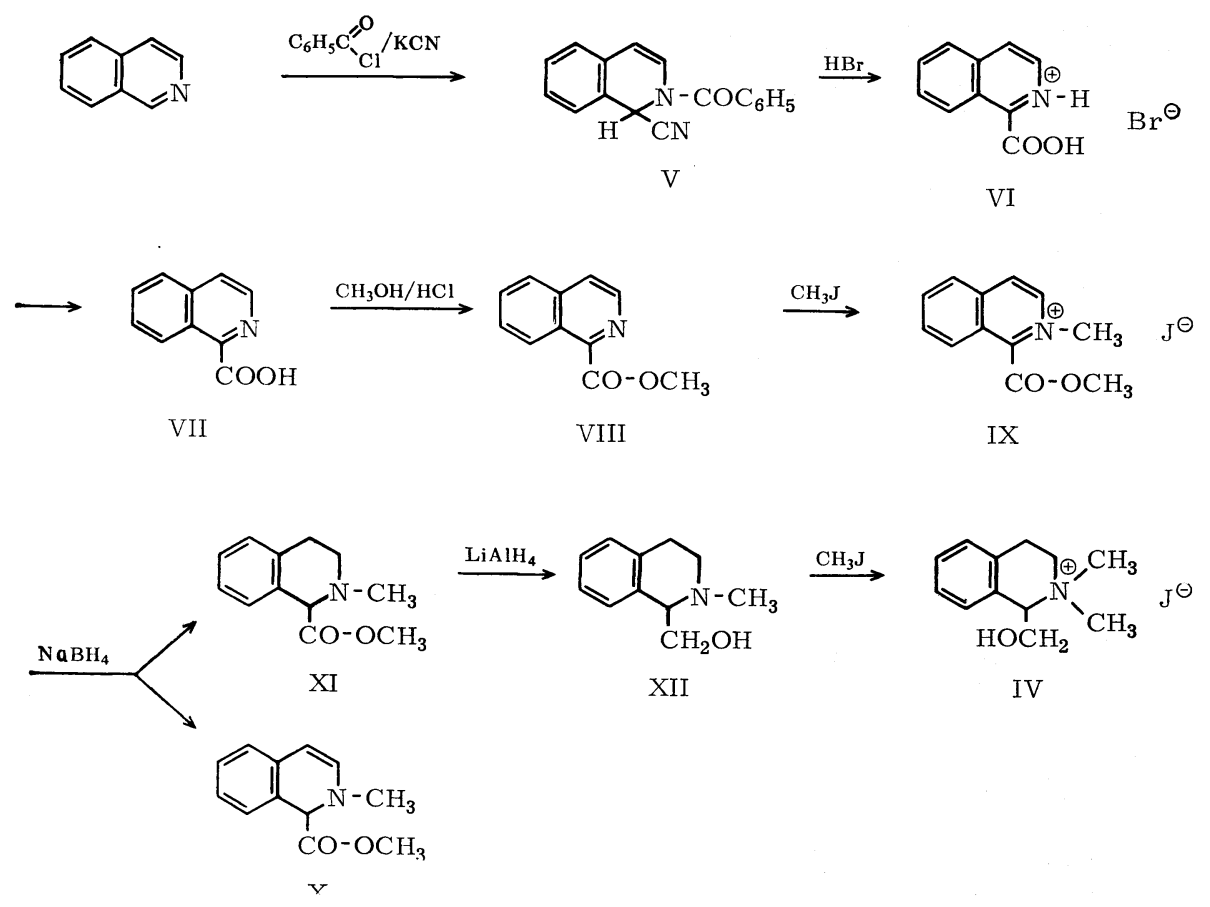

1-Cyan-2-benzoyl-1,2-dihydro-isochinolin $\mathrm{V}$ wurde nach Reissert ${ }^{4}$ ) hergestellt. Isochinaldinsäure VII läßt sich nach Reissert ${ }^{4}$ ) trotz erheblichen Arbeitsaufwandes nur in mäßigen Ausbeuten gewinnen. Durch Verseifen von V mit Bromwasserstoffsäure (vgl. $\left.{ }^{5}\right)$ ) konnten wir die Ausbeute zwar nur unwesentlich vergrößern, der Arbeitsaufwand ist jedoch, gemessen an dem Reissertschen Verfahren ${ }^{4}$ ), erheblich geringer. - Die Quaternisierung des Esters VIII war schwierig, die Umsetzung läuft auch bei langen Reaktionszeiten nicht quantitativ ab; nicht umgesetztes VIII kann jedoch zurückgewonnen werden. - Bei der Reduktion des N-Methyljodides IX mit Natriumborhydrid entstanden zu etwa gleichen Teilen ein 1,2-Dihydro-N-alkylisochinolin-Derivat - vermutlich X - und das tetrahydrierte Produkt XI. X wurde an seiner Reduktionswirkung gegenüber methanolischer Silbernitratlösung erkannt. Diese Reaktion läßt sich auch zum Nachweis im DC verwenden ${ }^{6}$ ). Eine Reduktion von IX zu XI mit Zink in Säure wurde nicht versucht, um die Estergruppe nicht anzugreifen. $\mathrm{X}$ zersetzte sich schnell und wurde nicht weiter untersucht.

Beim Hofmann-Abbau der quartären Base von IV mit Lauge fanden wir II und Formiat, das durch Cannizzarosche Reaktion aus Formaldehyd entstanden war.

4) A. Reissert, Ber. dtsch. chem. Ges. 38, 3427 (1905).

5) J. W. Davis jr., J. org. Chemistry 24, 1691 (1959).

$\left.{ }^{6}\right) E$. Roesel, unveröffentl. Versuche, Braunschweig 1967. 
II wurde durch de Vergleich mit authentischer Substanz und als N-Methyljodid identifiziert ${ }^{7}{ }^{1}$ ); Formiat wurde im Mikrotest ${ }^{8}$ ) durch die Reduktion von Quecksilber(II)-chlorid-Lösung nachgewiesen.

Dieses Experiment zeigt, daß auch in $\mathrm{N}$-quartären primären Carbinolen vom Typ IV beim Hofmann-Abbau der Substituent am C-1 abgespaiten wird.

Dem Land Niedersachsen sind wir für die Förderung dieser Arbeit durch Forschungsmittel zu Dank verpflichtet.

\section{Beschreibung der Versuche}

1-Cyan-2-benzoyl-1,2-dihydro-isochinolin $V$ wurde nach Lit.-Angaben hergestellt $\left.{ }^{4}\right)^{1}$ ).

Isochinaldinsäure (VII)

$30 \mathrm{~g} \mathrm{~V}$ wurden in $30 \mathrm{ml}$ Eisessig suspendiert und portionsweise mit insgesamt $36 \mathrm{ml}$ Bromwasserstoffsäure $(d=1,48)$ versetzt. Der Ansatz wurde anschließend 30 Min. unter Rückfluß erhitzt. Beim Abkühlen kristallisierte das Hydrobromid der Isochinaldinsäure (VI) aus. Es wurde abgesaugt, mit Eisessig und anschlitßend mit Äther gewaschen. Das Kristallisat wurde i. Vak. über Natriumhydroxid getrocknet. Schmp. 202-203 ${ }^{\circ}$. Ausbeute: $21 \mathrm{~g}$ $=77 \% \mathrm{~d}$. Th.

$21 \mathrm{~g}$ VI wurden in $50 \mathrm{ml}$ heißem Wasser gelöst und mit 0,5 g Kohle versetzt. Nach dem Filtrieren wurde die bräunlich gefärbte Lösung mit $15 \mathrm{ml}$ Ammoniak versetzt und das ausgefallene Ammoniumsalz durch Erhitzen wieder in Lösung gebracht. Die heiße Lösung wurde nochmals mit Kohle behandelt und filtriert. Nach Zugabe von $15 \mathrm{ml}$ Eisessig und Abkühlen auf Raumtemp. wurde die Lösung mit Chloroform mehrfach ausgeschüttelt. Isochinaldinsäure VII kristallisierte beim Einengen aus. Schmp. $162^{\circ}\left(\right.$ Lit. $\left.^{4}\right)$ : $161-162^{\circ}$ ). Ausbeute: $8,1 \mathrm{~g}=40 \%$ d. Th. (ber. auf V).

Isochinaldinsäuremethylester (VIII)

9,0 g VII wurden in $120 \mathrm{ml}$ Methanol auf dem Wasserbad gelöst; nach Sättigung der Lösung mit $\mathrm{HCl}$ wurde der Ansatz 4 Std. unter Rückfluß erhitzt. Nach 2 Std. wurde nochmals $\mathrm{HCl}$ eingeleitet. Nach dem Abkühlen wurde das Methanol i. Vak. abdestilliert, der Rückstand in Wasser aufgenommen, die wäßrige Lösung mit 10proz. Ammoniak alkalisiert und der Ester mit Äther ausgeschüttelt. Nach dem Trocknen und Abdestillieren des Lösungsmittels resultierte ein braunes Öl, welches im Kugelrohr bei 4 Torr und $110^{\circ}$ (Badtemp.) destilliert wurde. Ausbeute: $7 \mathrm{~g}=71 \%$ d. Th. Pikrat aus Äthanol,Schmp. $147^{\circ}$.

$$
\begin{array}{lll}
\mathrm{C}_{11} \mathrm{H}_{10} \mathrm{NO}_{2} \cdot \mathrm{C}_{6} \mathrm{H}_{2} \mathrm{~N}_{3} \mathrm{O}_{7}(416,3) & \text { Ber.: C 49,05 } & \mathrm{H} 2,91 \\
& \text { Gef.: C 49,13 } & \mathrm{H} 3,26
\end{array}
$$

Isochinaldinsäuremethylester-N-methyljodid (IX)

7,0 g VIII wurden in $40 \mathrm{ml}$ Aceton aufgenommen, mit $7 \mathrm{ml}$ Methyljodid versetzt und 10 Std. auf dem Wasserbad am Rückflußkühler erhitzt. In der Kälte kristallisierte das N-Methyljodid in gelben Plättchen. Es wurde aus Aceton/Methanol (3:1) umkristallisiert. Schmp. $150-151^{\circ}$. Ausbeute $4,0 \mathrm{~g}=32,7 \% \mathrm{~d}$. Th.

$$
\begin{array}{lll}
\mathrm{C}_{12} \mathrm{H}_{12} \mathrm{NO}_{2} \mathrm{~J}(329,1) & \text { Ber.: C } 43,79 & \mathrm{H} 3,68 \\
& \text { Gef.: C } 43,79 & \mathrm{H} 3,58
\end{array}
$$

7) H. Emde, Liebigs Ann. Chem. 391, 101 (1912).

8) F. Feigl, Spot Tests, Organic Applications, 4. Aufl., Elsevier Publishing Company, Amsterdam 1954, S. 246. 
N-Methyl-1,2,3,4-tetrahydro-isochinaldinsäuremethylester (XI)

$4 \mathrm{~g}$ IX wurden in $60 \mathrm{ml}$ Methanol gelöst und mit Natriumtetrahydridoboranat in kleinen Anteilen versetzt. Nach lstdg. Erhitzen der Reaktionslösung auf dem Wasserbad wurde das Methanol i. Vak. abdestilliert und die Base mit Äther ausgeschüttelt. Das Tetrahydroisochinolin-Derivat wurde von dem ebenfalls entstandenen 1,2-Dihydroisochinolin-Abkömmling durch präp. DC getrennt. Kieselgel $\mathrm{PF}_{254}$, ,Merck“. Fließmittel: Benzol 70, Aceton 25, Methanol 5. Ausbeute: $600 \mathrm{mg}$ einer öligen Verbindung. Pikrat: Schmp. 160 bis $161^{\circ}$ (aus Äthanol).

$$
\mathrm{C}_{12} \mathrm{H}_{16} \mathrm{NO}_{2} \cdot \mathrm{C}_{6} \mathrm{H}_{2} \mathrm{~N}_{3} \mathrm{O}_{7}(434,3) \quad \text { Ber.: } \mathrm{C} 49,78 \quad \mathrm{H} 4,18
$$

1-Hydroxymethyl-2-methyl-1,2,3,4-tetrahydro-isochinolin (XII)

$600 \mathrm{mg}$ XI wurden in $10 \mathrm{ml}$ abs. Tetrahydrofuran gelöst und zu einer Suspension von

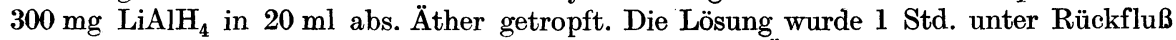
auf dem Wasserbad erhitzt. Unter Eiskühlung wurde mit Äther verdünnt und mit Wasser zersetzt. Die organische Phase wurde vom Niederschlag abgegossen, der Rückstand mit Tetrahydrofuran mehrfach gewaschen. Die Lösungsmittel wurden i. Vak. abdestilliert. Ausbeute: $450 \mathrm{mg}$ helles Öl. Pikrat: Schmp. 153 (aus Äthanol).

$$
\begin{array}{lll}
\mathrm{C}_{11} \mathrm{H}_{16} \mathrm{NO} \cdot \mathrm{C}_{6} \mathrm{H}_{2} \mathrm{~N}_{3} \mathrm{O}_{7}(406,3) & \text { Ber.: C 50,25 } & \mathrm{H} \mathrm{4,47} \\
& \text { Gef.: C 50,52 } & \mathrm{H} \mathrm{4,30}
\end{array}
$$

1-Hydroxymethyl-2-methyl-1,2,3,4-tetrahydro-isochinolin-N-methyljodid (IV)

$450 \mathrm{mg}$ Carbinol XII wurden in $5 \mathrm{ml}$ Aceton aufgenommen und mit $1 \mathrm{ml}$ Methyljodid versetzt. Es schieden sich sogleich Kristalle ab. Nach weiterer Zugabe von $15 \mathrm{ml}$ Aceton wurde noch 15 Min. auf dem Wasserbad unter Rückfluß erwärmt. Umkristallisation aus Aceton. Schmp. $195^{\circ}$. Ausbeute: $600 \mathrm{mg}$.

$$
\begin{array}{lll}
\mathrm{C}_{12} \mathrm{H}_{18} \mathrm{NOJ}(319,2) & \text { Ber.: } \mathrm{C} \mathrm{45,16} & \mathrm{H} 5,58 \\
& \text { Gef.: C } 45,27 & \mathrm{H} 5,56
\end{array}
$$

Hofmann-Abbau von IV

$0,5 \mathrm{~g} \mathrm{IV}$ wurden in $30 \mathrm{~m}$ ] Wasser von $35^{\circ}$ gelöst und am stark basischen Ionenaustauscher Merck III in die quartäre Base überführt. Das Elut wurde i. Vak. (Dampftemp. $30^{\circ}$ ) auf $10 \mathrm{ml}$ eingeengt, die Lösung mit $30 \mathrm{ml} 40 \mathrm{proz}$. $\mathrm{KOH}$ versetzt und 1 Std. auf dem siedenden Wasserbad erhitzt. Nach dem Erkalten wurde mit $50 \mathrm{ml}$ Wasser verdünnt. mit Äther ausgeschüttelt, die Ätherphase getrocknet und abdestilliert. Das zurückbleibende Öl wurde als N,N-Dimethyl-2-vinyl-benzylamin identifiziert. Es zeigte im DC gleiche Rf-Werte wie authentisches Material $\left.{ }^{7}\right)^{1}$ ).

In der wäßrigen Phase wurde im Mikrotest $\left.{ }^{8}\right)$ Ameisensäure nachgewiesen. II wurde in $5 \mathrm{ml}$ Äther aufgenommen und mit 0,5 ml Methyljodid versetzt. Es bildete sich bei Raumtemp. ein kristalliner Niederschlag, der aus Äthanol umkristallisiert wurde. Schmp. $201^{\circ}$ (Lit. ${ }^{7}$ ) : $199-200^{\circ}$ ), im Mischschmp. mit authentischem ${ }^{1}$ ) II-N-Methyljodid keine Depression.

\section{Allgemeine Angaben}

Die Schmp. wurden im Kupferblock bestimmt und sind unkorrigiert angegeben. Die Elementaranalysen wurden von Herrn M. Beller, Göttingen, durchgeführt. 